\title{
Ileus: Radiological Approach in Daily Practice
}

\author{
Hülya Kurtul Yıldız', Elif Evrim Ekin', Aylin Hasanefendioğlu Bayrak', İsmail Sinan Duman'1, Samed Sayar², Gökhan Tolga Adaş² \\ ${ }^{1}$ Clinic of Radiology, Gaziosmanpaşa Taksim Training and Research Hospital, İstanbul, Turkey \\ ${ }^{2}$ Clinic of Surgery, Gaziosmanpaşa Taksim Training and Research Hospital, İstanbul, Turkey
}

\begin{abstract}
lleus is defined as the inability to remove gut contents. Intestinal obstruction, the passage of intestinal contents is partially or completely blocked. The main target in ileus is to diagnose strangulation, which requires emergency surgery. Clinical findings, patient history, and X-ray are helpful in diagnosis. Computed tomography is very helpful in the differential diagnosis. The differential diagnosis of ileus and radiological findings are presented.
\end{abstract}

Keywords: Ileus, strangulation, computed tomography, bezoar, MRI enterography

Ileus is the inability to remove gut contents. In intestinal obstruction, the distal passage of intestinal content is partially or completely blocked.

The main goal in ileus is to establish the diagnosis of strangulation, which requires emergency surgery (1). Hernia, volvulus, and intussusception have a role in the etiology of strangulation. Diagnosis is established based on clinical findings, patient's anamnesis, and standing abdominal x-ray findings (1). Computed tomography $(\mathrm{CT})$ is highly useful in the differential diagnosis. Abdominal CT should be preferred over conventional barium graphs because barium suspension use can convert a partial bowel obstruction into a complete obstruction in retrograde passage graphs.

In the diagnosis of ileus, sensitivity of the standing abdominal $x$ ray varies from $48 \%$ to $80 \%$ (2). On the other hand, sensitivity and specificity of CT scans have been reported to be $93 \%$ and $100 \%$, respectively.

Due to obstruction, luminal pressure elevates and high pressure causes the rupture of small veins and venous/arterial failure. Intraabdominal pressure increases, and respiration and venous circulation are inhibited. Bacteria in the small intestine, which is almost sterile, rapidly increase, and bacterial translocation occurs. Mortality rate associated with sepsis/shock is $70 \%$. In a simple obstruction, the bowel is obstructed at a point, and some complaints including constipation, nausea, vomiting, colicky abdominal pain, and abdominal distension develop in patients. "Closed-loop" or strangulation occurs due to the occlusion of the bowel loop at two adjacent points. Fever, peritoneal irritation, tachycardia, leukocytosis, and metabolic acidosis are observed in such patients.

\section{How to Perform CT in Ileus?}

It is necessary to use intravenous (IV) contrast. If a patient can take fluid orally, oral contrast must be used. In the presence of a suspected intestinal hematoma in a patient receiving antico- agulant therapy, the use of IV contrast and sections without oral contrast will be useful in the evaluation of the hematoma in the intestinal wall, which has a high density.

\section{Findings of Ileus CT}

In ileus of the small bowel, the overall diameter of the small bowel segment is significant when it is above $2.5 \mathrm{~cm}$ (3). Colonic segments and obstruction distal are protected. Hemorrhage in the bowel wall due to obstruction or increased thickness of the bowel wall due to edema can be observed ( $>2 \mathrm{~mm}$ ).

In ileus of the large bowel, both the colon and small bowel segments are affected. The location of the obstruction should be investigated through segmental evaluation from the distal to the proximal ends.

Whether the obstruction is severe or partial can be determined according to the degrees of dilatation at the proximal end and collapse at the distal end. In severe obstruction, there is a diameter difference of $50 \%$ between the collapsed bowel loop at the distal end and dilated bowel loop at the proximal end. Moreover, the distal passage of the oral contrast agent according to the severity of the obstruction is another useful finding. Small bowel feces signs observed in the passage zone are also important.

All patients presented in this article are adults. The study does not include a population of children. Patients were admitted to the emergency service of our hospital between May 2014 and January 2016, and they were evaluated at the clinic of general surgery. With a prediagnosis of ileus, radiological examinations were performed, and it was confirmed via surgical findings.

Classification according to the etiology of ileus:

A) Mechanical ileus

B) Ileus associated with insufficient bowel motility (Paralytic ileus)

C) Pseudo-obstruction (Ogilvie's Syndrome) 


\section{A) Mechanical lleus}

\section{1- lleus of the small bowel:}

\subsection{Intraluminal Factors}

1.1.a. Intussusception (invagination): It is a rare cause of ileus found among adults $(<5 \%)$. The most common cause is neoplasia, adhesion, and a foreign body $(4,5)$.

1.1.b. Volvulus: It is defined as the torsion of the mesenteric tissue providing the vascular supply (Figure 1). As a result, an increase in wall thickness and density, halo or target sign, pneumatosis of the intestine, and the lack of wall stain can be detected.

1.1.c. Gallstone ileus, bezoar: A gallstone often leads to obstruction at the ileocecal junction with the fistula from the biliary tract.

Bezoar is a rare cause of ileus, and it is the accumulation of undigested bowel content. This should be kept in mind, particularly for patients with ileus due to foreign bodies in the stomach (Figure 2).

\subsection{Transluminal Factors}

\section{2.a. Inflammatory diseases: Ulcerative colitis, Crohn's disease}

Ulcerative colitis; Backwash ileitis is an inflammatory bowel disease that often involves the rectum and the entire colon and terminal ileum. It is mostly seen among men in the age group of 15-40 years with extraintestinal findings, and an increase of $0.5 \%-1 \%$ in the risk of malignancy can occur in chronic patients having this disease for 10 years or longer. Thickening in the haustras, pseudopolyp, stricture, abscess, and perforation can be observed on CT scans.

Crohn's disease; It can affect any part of the gastrointestinal tract. It develops between the ages of 15 and 25 years in both sexes at equal rates, and it presents with a course of skip lesions and ulcers. In long-term involvement, some complications such as structural obstruction, fistula, and abscess can be revealed through CT (Figure 3). In particular, some findings such as submucosal fat deposition, perivascular inflammation, contrast uptake in the intestinal wall, and increased wall thickness in the terminal ileum can be observed. All CT findings can also be evaluated through MR enterography, which is another advantageous modality owing to the absence of radiation and easy use in activation.

1.2.b. Neoplasia: Primary neoplasia of the small bowel is rare $(<2 \%)(6,7)$. Adenocarcinoma is observed more frequently. Metastatic involvement is more common than primary cancer (7).

1.2.c. Radiation enteritis, hematoma: Radiation enteritis occurs 1 year after radiotherapy, and ileal loops are mostly affected $(8$, 9). Hematoma is often seen in patients receiving anticoagulant treatment or secondary to trauma.

1.2.d. Vascular causes: It develops in association with mesenteric artery or vein occlusion. Artery occlusion is more common than vein occlusion. Although catheter angiography is considered the gold standard for diagnosis, CT is valuable because it is easier to reach and is a rapid technique. It can show mesenteric veins and affected bowel loops with thickened walls that demonstrate asymmetric contrast (Figures 4, 5).
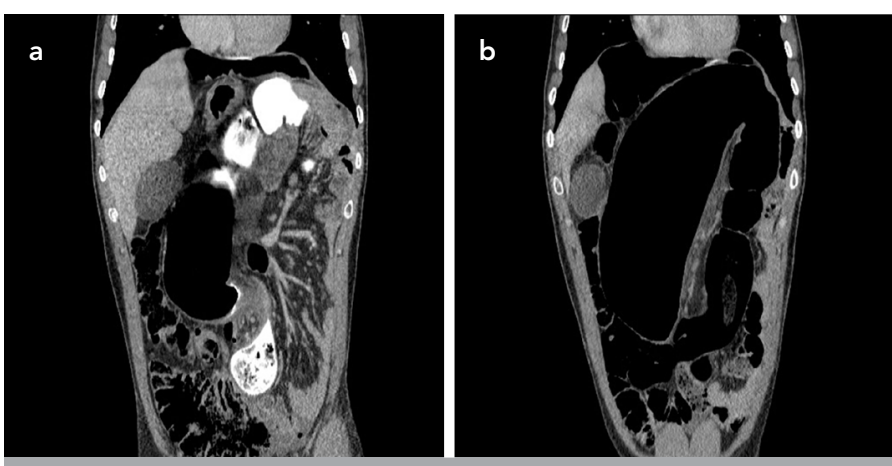

Figure 1. a, b. A 57-year-old female patient with volvulus in the small intestine. (a) Whirl sign is observed in the coronal plane of computed tomography (CT) from the midline of the abdomen. (b) Highly expanded intestinal segments that are full of air are viewed in the coronal CT section.
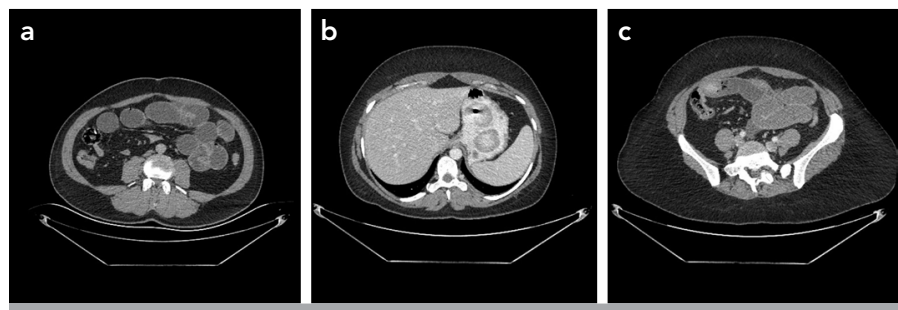

Figure 2. a-c. A 50-year-old male patient. (a) Expanded small bowel segments and intraluminal bezoar densities are viewed in the axial computed tomography section. (b) In another male patient at the age of 36 years, the densities of a round foreign body are observed in the stomach. (c) In a similar view, bezoar densities are included in the distal ileal segment, and they cause ileus. Both cases were confirmed with surgical findings.
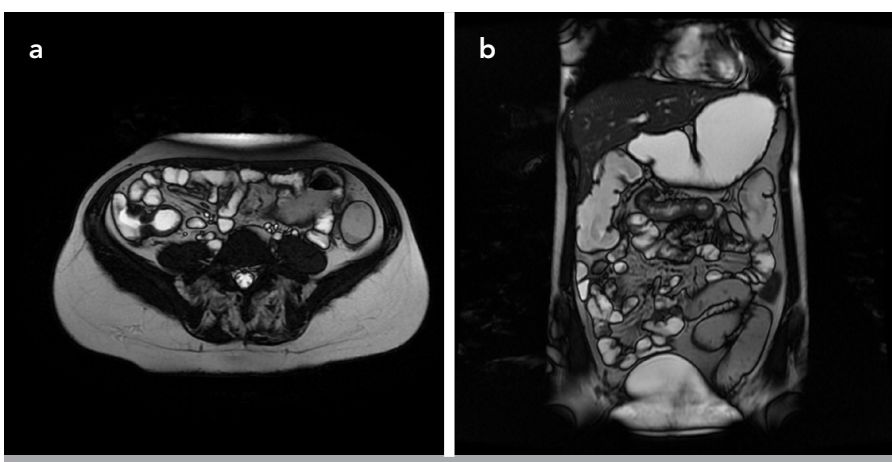

Figure 3. a, b. A 30-year-old female patient diagnosed with Crohn's disease. (a) In T2-weighted axial magnetic resonance (MR) enterography, a small bowel obstruction associated with terminal ileitis and multiple staging is viewed. (b) Thickening was found in the jejunal mucosa in T2-weighted coronal MR.

1.2.e. Congenital atresia-stenosis and postoperative obstructions (Figure 6).

1.2.f. Meckel's diverticulum: Meckel's diverticulum is the most common congenital anomaly of the intestinal canal, and its incidence is $2 \%$. It develops because of incomplete regression of the omphalomesenteric canal. It is mostly seen at any point of the ileocecal junction, and it is $100 \mathrm{~cm}$ proximal (75\%) (10). It is generally asymptomatic, and the rate of its lifelong complications has been reported to be $4 \%-10 \%$ (11). In symptomatic patients, het- 


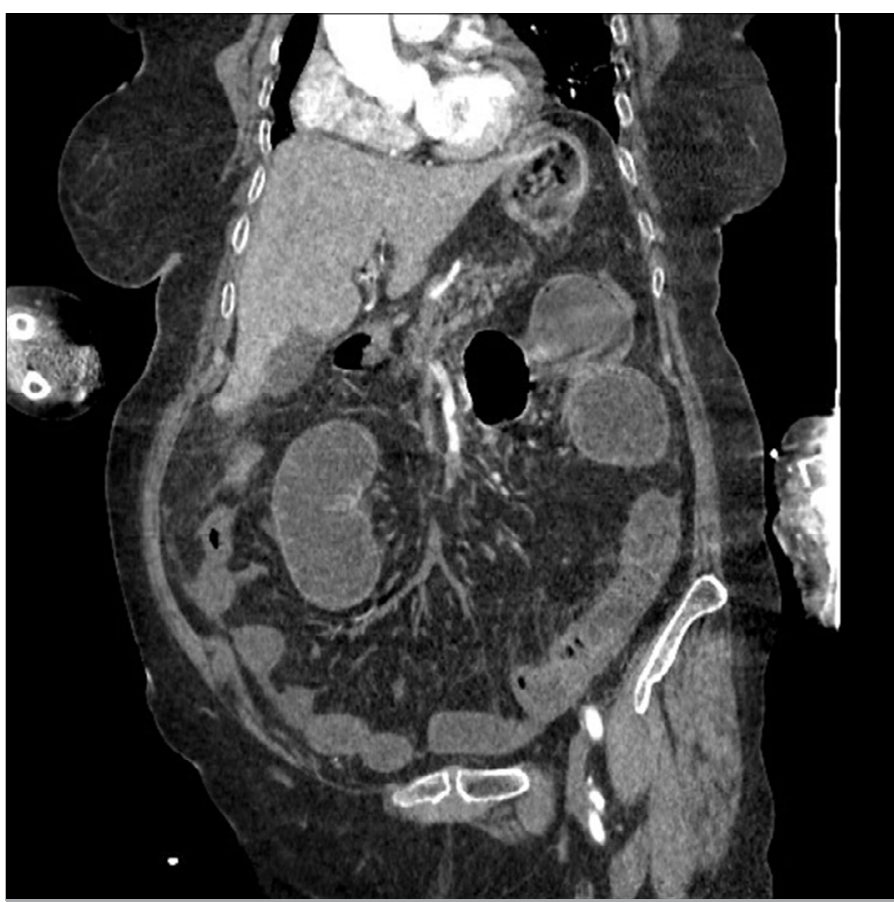

Figure 4. In a 78-year-old male patient, the contrast filling defect associated with thrombus and thrombus material is viewed in the superior mesenteric artery in the coronal plane computed tomography examination.
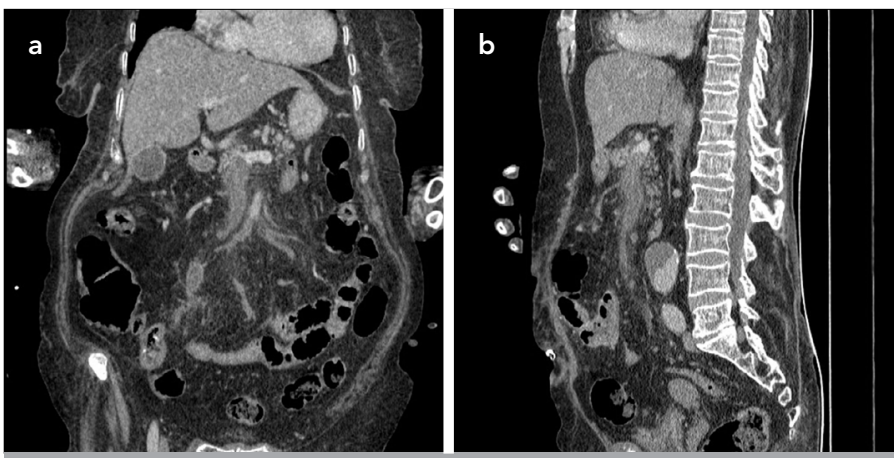

Figure 5. a, b. An 80-year-old female patient. (a) In coronal computed tomography (CT) and (b) sagittal CT sections, thrombus filling and expanding the lumen were found in the superior mesenteric vein and portal vein.

erotopic gastric and pancreatic mucosa are histologically found in Meckel's diverticulum (11). The most frequently seen complications of Meckel's diverticulum are hemorrhage, obstruction, and diverticulitis (12).

\subsection{Extraluminal Factors}

1.3.a. Adhesions: It is the most common cause of small bowel obstruction (50\%-80\%) (13). It mostly develops secondary to previous abdominal surgery. The adhesion band cannot be clearly viewed through CT. It is necessary to eliminate other pathologies for the diagnosis of bride ileus. Sudden changes in the diameter of the small bowel loop in CT helps establish a diagnosis (Figure 7).

1.3.b. Hernia: It is the second most common cause of ileus (14). In daily practice, we can encounter ileus associated with small

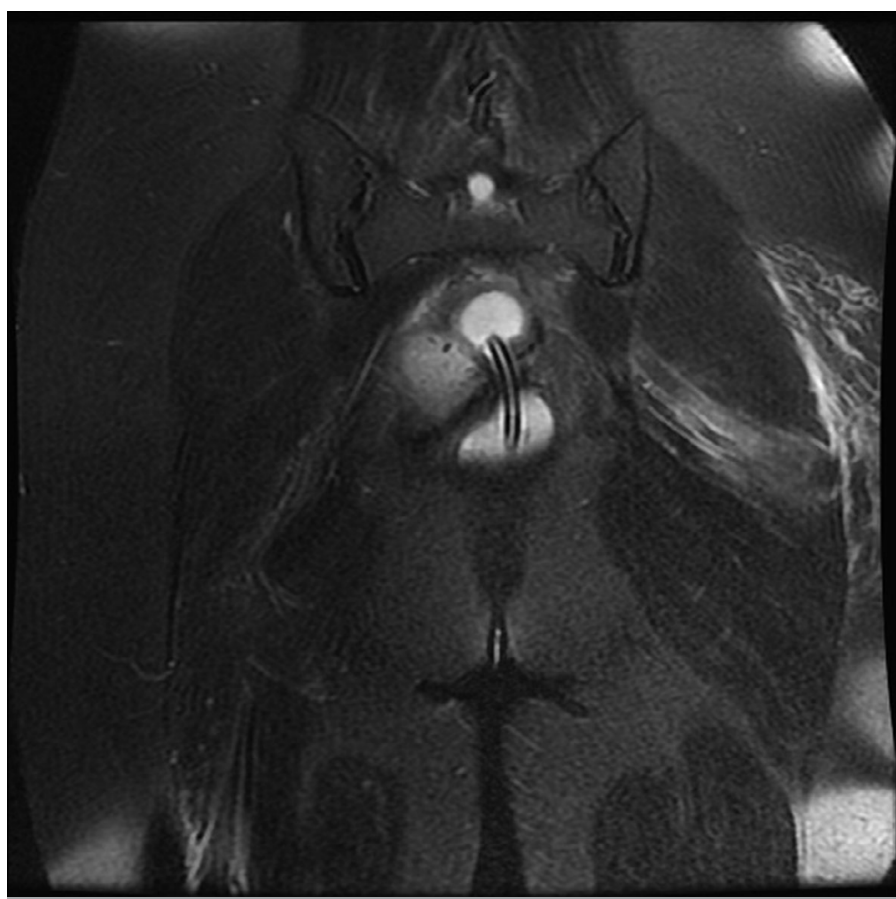

Figure 6. In a 46-year-old female patient, an obstruction of approximately $2 \mathrm{~cm}$ in length in the surgical site that developed after the operation of rectum adenocarcinoma; coronal fat-saturated T2weighted magnetic resonance section.

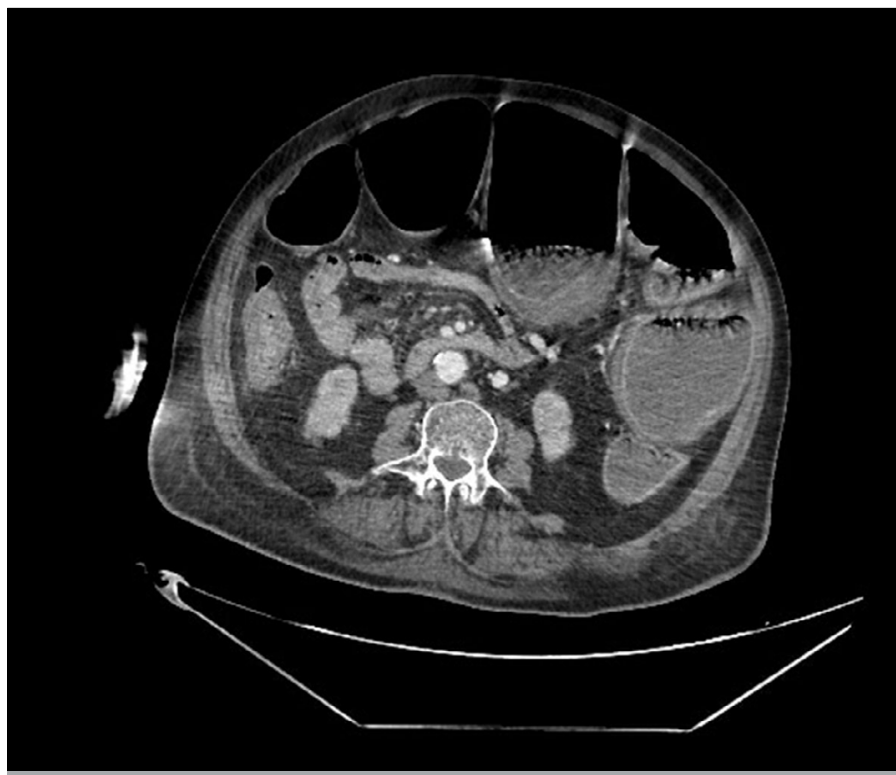

Figure 7. In a 55-year-old male patient, adhesion-induced small bowel obstruction. In the axial computed tomography section from the level of umbilicus, multiple air fluid levels, known as beak sign, and the sudden passage zone are shown.

bowel loops protruded in inguinal hernia and incisional hernia. In the presence of hernia in patients with ileus, bowel segments should be investigated in hernial sac. If there is intestinal distension in the proximal of the hernial level, it indicates ileus (Figures 8,9$)$.

Internal hernia: It is a rare pathology, and its frequency is between $0.5 \%$ and $5.8 \%$. The most common types are para- 


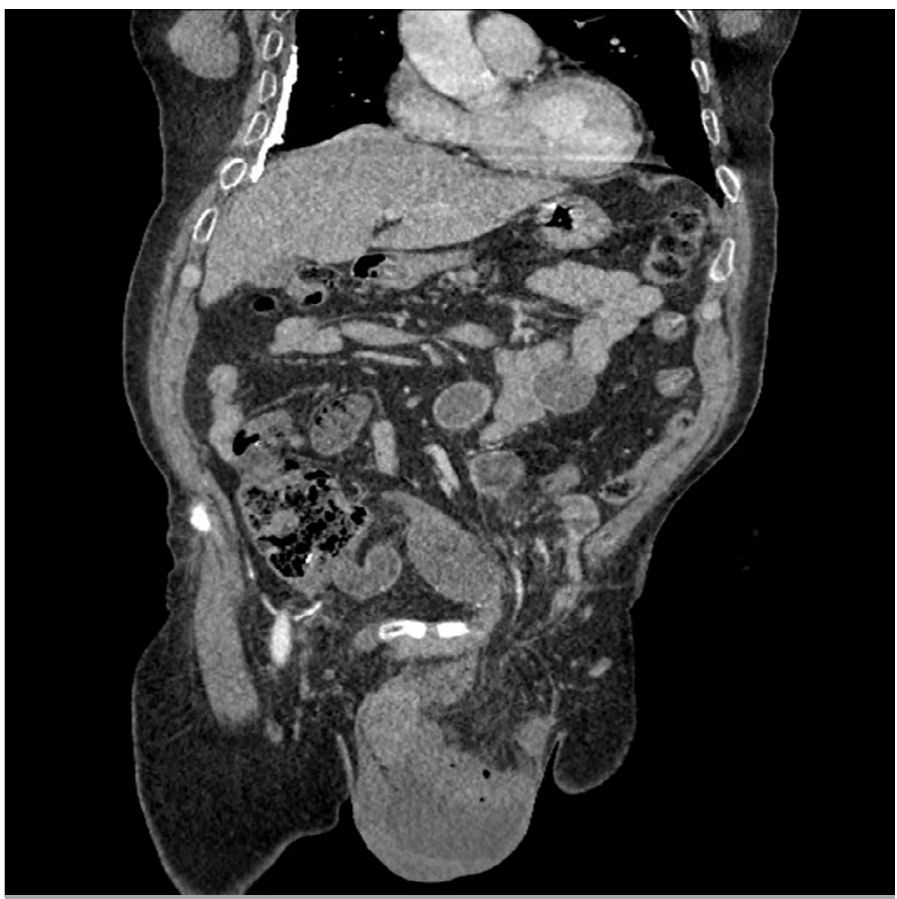

Figure 8. In a 74-year-old male patient, small bowel obstruction due to incarcerated left inguinal hernia. Incarcerated bowel loop with thickened wall in the left inguinal canal was detected in the coronal computed tomography section.

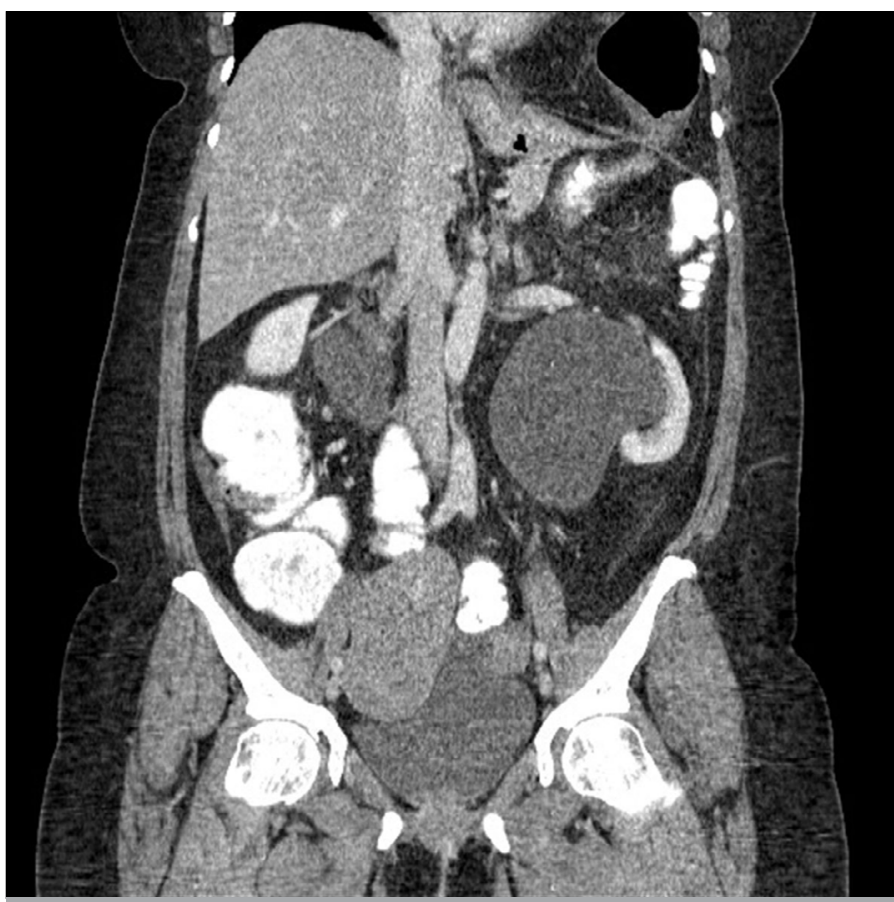

Figure 9. In a 55-year-old male patient, herniated splenic flexure extending from the left hemidiaphragmatic defect to the left hemithorax was observed. Additionally, dilatation was detected in the proximal loops.

duodenal hernia (53\%) and omental foraminal hernia (8\%). CT plays an important role in the detection of the unusual position of a hernial sac, including in small bowel segments (Figure 10).

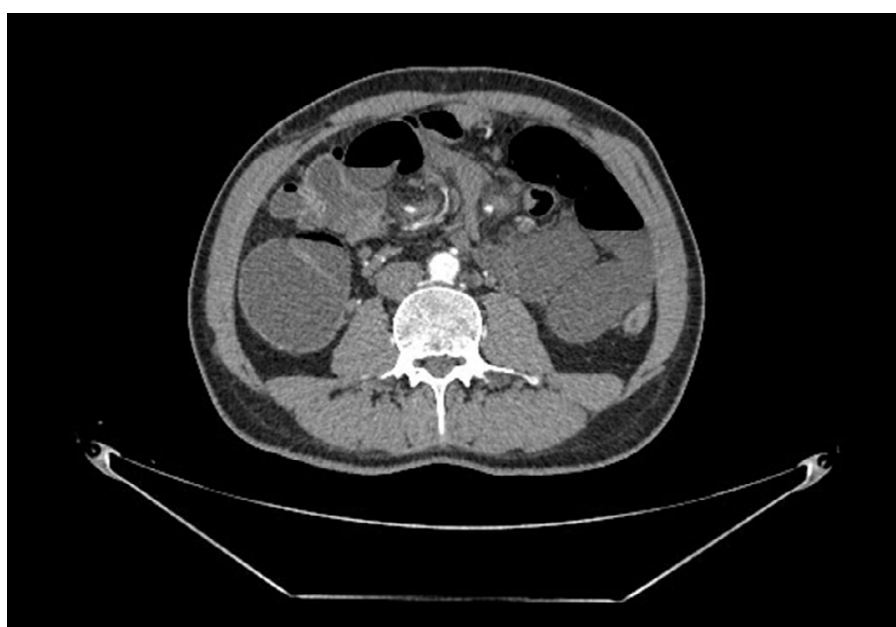

Figure 10. Omental foraminal hernia in a 68-year-old male patient; atypical configuration of the intestinal loop passing from the midline defect of the abdomen in the axial computed tomography section and ileus of the small bowel were detected.

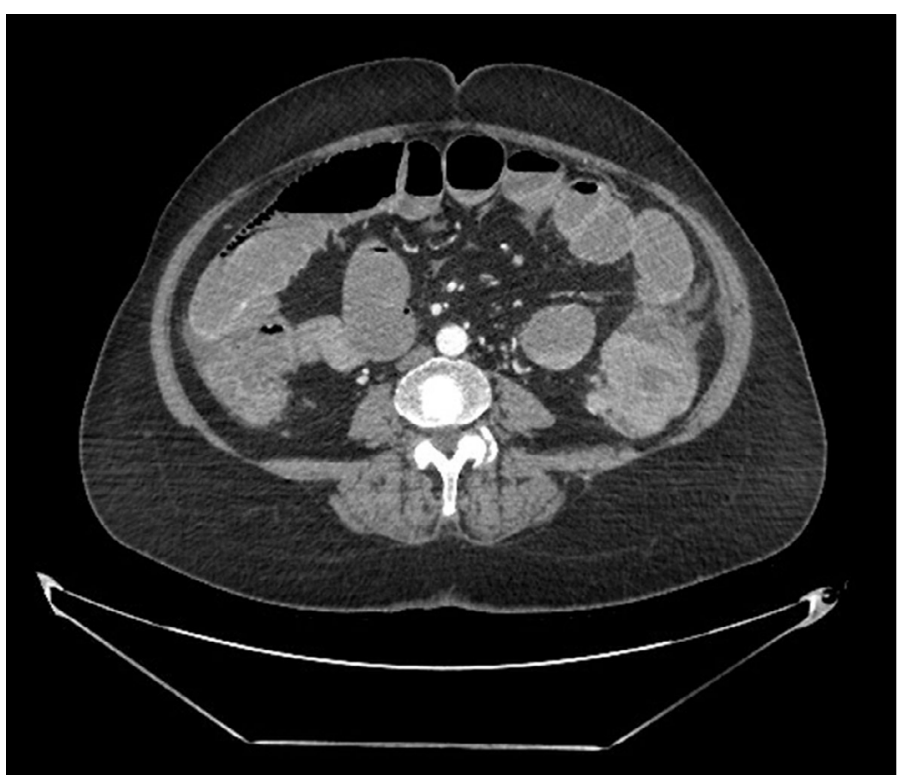

Figure 11. In a 60-year-old female patient with acute abdominal pain, irregular and solid masses with heterogeneous structures in the sigmoid colon and wide bowel loops were detected in the axial computed tomography section.

1.3.c. Intraabdominal masses, peritonitis carcinomatosa (the most common cause is ovarian neoplasia): They induce extrinsic bowel obstruction.

\section{2. lleus of the Large Bowel}

The main etiological factor that causes ileus of the large intestine is neoplasia (Figure 11). In addition, inflammatory periods such as volvulus and diverticulitis can also lead to ileus.

\section{B) Ileus associated with insufficient bowel motility (Paralytic} ileus): It is mostly encountered in the acute postoperative period. Distension of both the colon and the small bowel is observed. 
C) Pseudo-obstruction (Ogilvie's Syndrome): It is defined as acute colonic pseudo-obstruction, and it is the diffuse distension of the colon in the absence of mechanical obstruction. Cecal perforation can develop due to untreated Ogilvie's syndrome. Nasogastric decompression, cessation of oral intake, and conservative treatment with electrolyte balance are the first choices in the treatment. Neostigmine is used for treatment (16).

\section{CONCLUSION}

It is usually possible to identify the etiology of ileus through a patient's anamnesis, clinical examination, standing abdominal x-ray, and CT. First of all, strangulation should be recognized. Then, the affected bowel segments should be examined, and a differential diagnosis should be made. Abdominal CT is guiding in the differential diagnosis of ileus.

Peer-review: Externally peer-reviewed.

Author Contributions: Concept - H.K.Y.; Design - E.E.E.; Supervision - A.H.B.; Resources - G.T.A.; Materials - G.T.A.; Data Collection and/or Processing - I.S.D., S.S.; Analysis and/or Interpretation - E.E.E.; Literature Search - H.K.Y.; Writing Manuscript - H.K.Y., E.E.E.; Critical Review - A.H.B.; Other - G.T.A.

Conflict of Interest: No conflict of interest was declared by the authors.

Financial Disclosure: The authors declared that this study has received no financial support.

\section{REFERENCES}

1. Silva AC, Pimenta M, Guimarães LS. Radiographics Small Bowel Obstruction: What to Look For? 2009; 29: 423-39.

2. Torreggiani WC, Harris AC, Lyburn ID, al-Nakshabandi NA, Zwirewich CV, Brenner $\mathrm{C}$, et al. Computed tomography of acute small bowel obstruction: pictorial essay. Can Assoc Radiol J 2003; 54: 93-9.

3. Fukuya T, Hawes DR, Lu CC, Chang PJ, Barloon TJ. CT diagnosis of small-bowel obstruction: efficacy in 60 patients. AJR Am J Roentgenol 1992; 158: 765-9. [CrossRef]
4. Agha FP. Intussusception in adults. AJR Am J Roentgenol 1986; 146 : 527-31. [CrossRef]

5. Boudiaf M, Soyer P, Terem C, Pelage JP, Maissiat E, Rymer R. CT evaluation of small bowel obstruction. Radiographics 2001; 21: 613-24. [CrossRef]

6. Qalbani A, Paushter D, Dachman AH. Multidetector row CT of small bowel obstruction. Radiol Clin North Am 2007; 45: 499-512. [CrossRef]

7. Mak SY, Roach SC, Sukumar SA. Small bowel obstruction: computed tomography features and pitfalls. Curr Probl Diagn Radiol 2006; 35 : 65-74. [CrossRef]

8. Furukawa A, Yamasaki M, Takahashi M, Nitta N, Tanaka T, Kanasaki S, et al. CT diagnosis of small bowel obstruction: scanning technique, interpretation and role in the diagnosis. Semin Ultrasound CT MR 2003; 24: 336-52. [CrossRef]

9. Deitel M, Vasic V. Major intestinal complications of radiotherapy. Am J Gastroenterol 1979; 72: 65-70.

10. Satya R, O'Malley JP. Case 86: Meckel diverticulum with massive bleeding. Radiology 2005; 236: 836-40. [CrossRef]

11. Fink AM, Alexopoulou E, Carty H. Bleeding Meckel's diverticulum in infancy: unusual scintigraphic and ultrasound appearances. Pediatr Radiol 1995; 25: 155-6. [CrossRef]

12. Levy AD, Hobbs CM. From the archives of the AFIP. Meckel diverticulum: radiologic features with pathologic correlation. Radiographics 2004; 24: 565-87. [CrossRef]

13. Delabrousse E, Destrumelle N, Brunelle S, Clair C, Mantion G, Kastler B. CT of small bowel obstruction in adults. Abdom Imaging 2003; 28: 257-66. [CrossRef]

14. Miller G, Boman J, Shrier I, Gordon PH. Etiology of small bowel obstruction. Am J Surg 2000; 180: 33-6. [CrossRef]

15. Martin LC, Merkle EM, Thompson WM. Review of internal hernias:radiographic and clinical findings. AJR Am J Roentgenol 2006; 186: 703-17. [CrossRef]

16. Maloney N, Vargas HD, Acute Intestinal Pseudo-Obstruction (Ogilvie's Syndrome) Clin Colon Rectal Surg 2005; 18: 96-101. [CrossRef] 